\title{
A kind of high precision synchronization algorithm for TR - UWB system Xiaoming Huang ${ }^{1, a}$, Guoliang Wang ${ }^{2, b}$

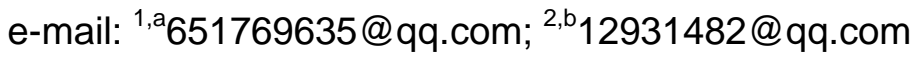 \\ Nanchang institute of science \& technology, China
}

Keywords: Ultra-wide-band communication, TR-UWB, Synchronous transmission, Simulation analysis

\begin{abstract}
The synchronization is the key problem of UWB system, because the non related systems of TR - UWB (Transmitted Reference Ultra - wide - band) evaded the problem of channel estimation, become a kind of hot technology. This paper put forward a kind of high precision synchronization algorithm, made the synchronization error within the set pulse width, greatly improved the ability to resist noise for communication receiver. Analyzed average capture time and capture probability of synchronized for TR - UWB systems, through the simulation experiment confirmed the effectiveness of algorithm.
\end{abstract}

\section{Introduction}

Ultra-wide-band radio uses nanosecond pulse with very low duty cycle to transfer information. Because the energy of pulse is low, each symbol use multiple narrow pulse transmission to obtain processing gain. The signal reception at the receiving end can be divided into coherent reception and non coherent reception. Coherent reception need accurate channel information and the design of receiving template signal, in order to get more signal. Non coherent receiver uses the receiving signal to do templates or do energy detection, which greatly reduces the complexity of the receiver. TR - UWB is a very popular solution which adopts non related auto-correlation receiver.

Coherent receiver must realize the synchronization of pulse level before realizing the symbol synchronization, then extract the symbol synchronization ${ }^{[6]}$. Non coherent receiver is the opposite, must go to realize the symbol synchronization, then guide the signal and information alignment. Most of the error of symbol synchronization algorithm in a frame range, it is not enough for demodulating the received signal, because of the low duty cycle and specific location of pulse in the frame is unknown, under the limit condition, can lead to the error rate is $50 \%$. However, due to the receiver with respect to the coherent receiver has good ability to resist synchronization error, synchronization of pulse level is unnecessary, because the cost is too high.

In order to make full use of the performance to resist synchronization error for non coherent receiver, this paper puts forward a kind of synchronization algorithm which precision is much less than the frame time. Making full use of the performance of TR-UWB system, done the precision can be set, can compromise between accuracy and capture time, in order to realize different function of the system ${ }^{[7]}$. Although achieved the similar function, but take the maximum delay $T_{m}$ of system as known parameter, in the real environment, $T_{m}$ is unable to predict in advance, therefore it doesn't have practicability.

\section{System model}

In order to facilitate the research, it is assumed that the signal system is binary, single user, the transmit signal is expressed as:

$S(t)=\sum_{i=0}^{\infty} \sum_{j=0}^{N_{f}-1}\left(\sqrt{E_{p}} p\left(t-i N_{f} T_{f}-j T_{f}\right)+L_{i} \sqrt{E_{p}} p\left(t-i N_{f} T_{f}-j T_{f}-D\right)\right.$

Among them, $N_{f}$ refers to the number of frame which frame no. is f, $T_{f}$ refers to the duration 
of data frame, $\left\{L_{i}= \pm 1\right\}$ refers to the bit sequence, $E_{p}$ refers to the signal pulse energy, $p(t)$ refers to the waveform of normalized pulse within the time $\mathrm{t}$, the duration is $T_{p}$, the duration of each symbol is $T_{s}=N_{f} T_{f}$, each frame transfers two pulses, the delay time is $D$, one is guide pulse, the second is the symbol which carried the information 。In order to avoid the pulses between the symbols, it is assumed that $T_{f} \geq 4 D$ in the process of research。

Ultra-wide-band channel adopts the model of distinguishable tapped delay: $h(t)=\sum_{l=0}^{N_{m}-1} \alpha_{l} \delta\left(t-T_{l}\right)$, $N_{m}$ is maximum resolution path number, channel amplitude parameter is a log normal distribution, effective multi-path delay is defined as: $T_{m}=T_{N_{m}-1}-T_{0}+T_{P}$. Usually, $T_{f}$ is much bigger than $T_{m}$, select $D>T_{m}+T_{p}$, in order to avoid the interference between intra-frame and inter-frame, the signal of receiver can be expressed as:

$$
\begin{aligned}
& r(t)=\sum_{i=0}^{\infty} \sum_{j=0}^{N_{f}-1}\left(\sqrt{E_{p}} g\left(t-i N_{f} T_{f}-j T_{f}-T_{0}\right)+L_{i} \sqrt{E_{p}} g\left(t-i N_{f} T_{f}-j T_{f}-T_{0}-D\right)+n(t)\right. \\
& g(t)=p(t) * h\left(t+T_{0}\right) \text {, the effective range of the signal is }\left[0, T_{m}\right] \text {, the mark of * in above formula }
\end{aligned}
$$
refers to convolution. $n(t)$ is additive Gaussian noise, average value is $0, N_{0} / 2$ refers to bilateral power spectral density. The signal bandwidth of band-pass filter which received source signal passed through is $w$, center frequency is $f_{0}$ 、 colored Gaussian noise signal is $\hat{n}(t)$, relation function is: $R_{\hat{n}}(T)=w N_{0} \frac{\sin (\pi w T)}{\pi w T} \times \cos \left(2 \pi f_{0} T\right)$ 。 Received signal $\gamma(t)$ after passed through band-pass filter expressed as $\hat{r}(t)$.

\section{Algorithm description}

(1) Symbol synchronization algorithm

Adopting the algorithm with data-aided $L_{i}=1$, the following information symbol $L_{i+1}=0$, information symbols are alternate, in order to get symbol synchronization information as soon as possible ${ }^{[3]}$.

It is assumed that receiver captures signal when $n=0$, selects the signal which symbol length is $2 \mathrm{~N}$, operation as below:

$$
I_{j}=\sum_{n=0}^{N_{f}-1} \int_{0}^{\frac{T_{f}}{2}} \hat{r}\left(t+n T_{f}+j \frac{T_{f}}{2}\right) \hat{r}\left(t+n T_{f}+j \frac{T_{f}}{2}+D\right) d t, j=0, \cdots, 2 N_{f}-1
$$

In the absence of noise, formula (3) is equivalent to comparing the energy contained in each symbol of $2 N_{f}$. Because of the adjacent two symbols are interactive, when the complete synchronization, $I_{j}$ must get the maximum value different symbols, the value of $I_{j}$ is canceled each other. Therefore, at the synchronous moment, $\hat{T}=\frac{T_{f}}{2} \arg \max _{j} I_{j}$ 。

(2) High-precision synchronization 
After the symbol synchronized, adopting training sequence of full 1, can according to the precision of the system, adopting the threshold comparison method, to realize the synchronization of high precision。It is assumed that the symbol is $T_{0}$, then to use $\left[-\frac{T_{f}}{2}+T_{0}, \frac{T_{f}}{2}+T_{0}\right]$ to calculate

$$
U=\int_{T_{v}-\frac{T_{f}}{2}}^{T_{v}+\frac{T_{f}}{2}} \hat{r}\left(t-T_{0}-T_{v}\right) \hat{r}\left(t-T_{0}-T_{v}-D\right) d t
$$

If $\mathrm{U}$ is less than threshold value $T_{1}$, then enter judgment of the next $T_{v}$, in order to fa cilitate the analysis, adopts the true method of random selection. In order to reduce the dis criminant error, in the phase of calibration and verification, can adopt the calculation of mu ltiple frames to improve the signal-to-noise ratio, it is assumed that the times of repetition $\mathrm{i}$ $\mathrm{n}$ the phase of calibration and verification is $G_{0}$ and $G_{1}$, then the time spend in the phase of calibration and verification is $G_{0} T_{f}$ and $G_{1} T_{f}$.

\section{Performance analysis}

In order to achieve the best performance, analyzing the average capture time of the syste $\mathrm{m}$, simplifying the process flow diagram, can use an expression to represent the closure of the capture time. The average capture time of symbol synchronization is very simple.

$$
E\left[T_{\text {symbol }}\right]=2 N_{f} T_{f}
$$

In order to improve the anti-interference ability, adopts method of repeating sampling sev eral times and calculating the average value. It is assumed that the average time is $k$, then

$$
E\left[T_{\text {symbol }}\right]=2 k N_{f} T_{f}
$$

High precision synchronization capture the signal flow chart as follows:

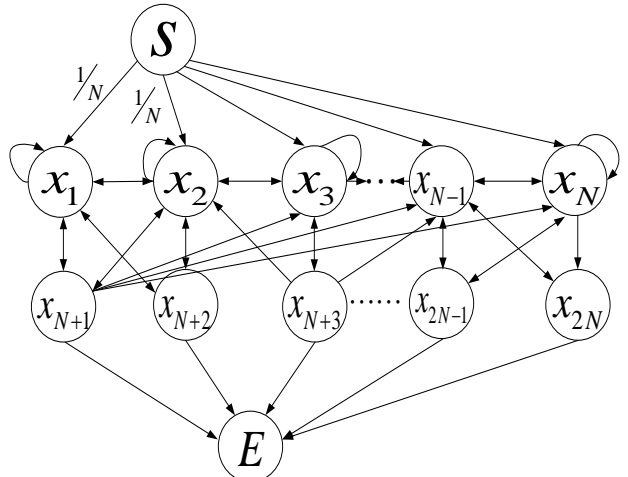

Figure1 Signal flow diagram

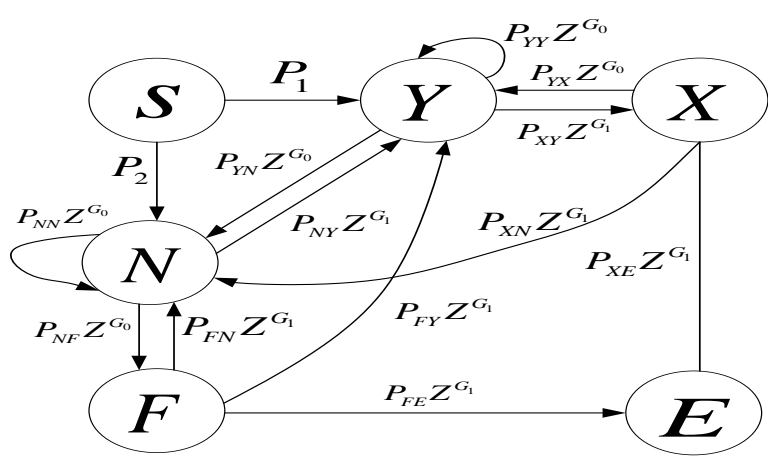

Figure2 Simplified flow diagram

Figure 1 can calculate state transfer function $H(z)$, through $H(z)$ can calculate the aver age capture time $\bar{T}=d H(z) / d z \mid z=1$. In order to get $H(z)$, we need to calculate transfer fu nction of each arrow, the simple channel is constant during the synchronization phase, the output of the integrator in formula (4) only is the function of $T_{v}$.

$$
U=\sqrt{E_{p}} R_{\hat{r}(t) \hat{r}(t)}\left(-T_{v}\right)+\hat{n}(t)
$$


$\bar{U}$ is Gaussian amplifier variable, average value is $E_{u}=\sqrt{E_{p}} R_{\hat{r}(t) \hat{r}(t)}\left(-T_{v}\right)$, variance is $\Delta^{2}=\sigma^{2} / 2 G$. The probability of inspection phase $x=T_{1}$ and verification phase $x=T_{2}$ can be obtained easily:

$$
P(\bar{U}>x)=\frac{1}{\sqrt{2 \pi}} \int_{x}^{+\infty} \frac{\left(x-E_{u}\right)}{\sigma / \sqrt{G}} e^{-\frac{x^{2}}{2}} d x
$$

$G$ refers to the times of signal repetition in inspection and verification phase.

Define $N=T_{f} / T_{v}$. The partition of $U>0$ is a valid partition, the partition of $U \leq 0$ is a invalid partition, define the valid partition as a collection $M$, define the invalid partition as a collection K, their number respectively defined as $N_{\text {Valid }}$ and $N_{\text {Null }}$. Obviously: $N=N_{\text {Valid }}+N_{\text {Null }}$. Complex flow diagram can be simplified as a state diagram expressed by the collection, as shown in figure 2.

Above figures simplified the complex signal process. Among them, $S$ is initial state collection, $Y$ is valid partition collection, $N$ is invalid partition collection, $E$ is termination state collection, $X$ is valid partition collection of inspection phase, $F$ is invalid partition collection of inspection phase. Obviously:

$P_{2}=N_{\text {Null }} / N$

Through deduction:

$$
P_{Y X}=\frac{1}{N_{\text {Valid }}} \sum_{i \in M} P_{X_{i} X_{i}+N} \quad \text { (10) } \quad P_{N F}=\frac{1}{N_{\text {Null }}} \sum_{i \in K} P_{X_{i} X_{i}+N}
$$

According to the theory of Markov chain, through deduction:

$$
\begin{aligned}
& P_{F E}=\frac{\sum_{i \in M} P_{X_{i} X_{i}+N} P_{X_{i}+{ }_{N} X_{E}}}{\sum_{i \in K} P_{X_{i} X_{i}+N}} \quad(12) \quad P_{X Z}=\frac{\sum_{i \in M} P_{X_{i} X_{i}+{ }_{N}} P_{X_{i}+N} X_{E}}{\sum_{i \in K} P_{X_{i} X_{i}+_{N}}} \\
& P_{X Y}=P_{1}\left(1-P_{X E}\right), P_{X N}=P_{2}\left(1-P_{X E}\right), P_{F Y}=P_{1}\left(1-P_{F E}\right), P_{F N}=P_{2}\left(1-P_{F E}\right)
\end{aligned}
$$

According to the simplified flow chart of figure 2, the application of Mason formula:

$$
\begin{aligned}
& H(z)=\frac{P_{F E} P_{N F}\left(P_{1} \beta+P_{2} Y\right)+P_{X E}\left(P_{1} \alpha+P_{2} \theta\right)}{\alpha \gamma-B \theta} \cdot Z^{G_{0}+G_{1}} \\
& \alpha=1-P_{N N} Z^{G_{0}}-P_{F N} P_{N F} Z^{G_{0}+G_{1}} \\
& \gamma=1-P_{Y Y} Z^{G_{0}}-P_{X Y} P_{Y X} Z^{G_{0}+G_{1}} \\
& \beta=P_{Y N} Z^{G_{0}}+P_{X N} P_{Y X} Z^{G_{0}+G_{1}} \\
& \theta=P_{N Y} Z^{G_{0}}+P_{F Y} P_{N F} Z^{G_{0}+G_{1}}
\end{aligned}
$$

The average capture time: $\quad \bar{T}=d H(z) / d z \mid z=1$

Because the probability of false alarm occurs during the inspection and verification phase, therefore, the average capture time is just one part of parameters for measuring system, and can't completely react the performance of system, the probability of capture is also an important 
parameter, most previous papers assume that the calibration system to identify the false alarm with probability 1, it is not possible in the real system, the flow diagram of Markov in figure 2, give full consideration to the probability of false alarm in inspection stage, using the $H(Z)$ and Markov theory obtained by the flow chart, we can obtain the expression of acquisition probability:

$$
\begin{aligned}
& P_{\text {detetion }}= \\
& \left|\frac{P_{F E} P_{N F}\left(P_{1} P_{\mathrm{IN}}+P_{1} P_{X N} P_{V X}+P_{2}-P_{2} P_{Y Y}-P_{2} P_{X Y} P_{\mathrm{VXX}}\right)+P_{X E} P_{V X}\left(P_{1}-P_{1} P_{N N}-P_{1} P_{N F} P_{F N}+P_{2} P_{N Y}+P_{2} P_{N Y}+P_{2} P_{F Y} P_{N F}\right)}{\left(1-P_{N N}-P_{F N} P_{N F}\right)\left(1-P_{Y Y}-P_{X Y} P_{V X}\right)-\left(P_{W N}+P_{X N} P_{V X}\right)\left(P_{N Y}+P_{F Y} P_{N F}\right)}\right|
\end{aligned}
$$

\section{Simulation and results}

In order to verify the validity of the algorithm, the second derivative of the Gaussian pulse is used for simulation. Basic parameters set as follows:

$$
N_{f}=10, T_{f}=100 \mathrm{~ns}, T_{p}=0.7 \mathrm{~ns}, N_{m}=15, T_{v}=3 T_{p}, E_{p} / N_{0}=10 \mathrm{~dB} \text {, use the fixed value }
$$

$G_{0}=3$ and $G_{1}=3$.

On the platform of Matlab, the figure of average capture time (unit: $\mathrm{T}_{\mathrm{s}}$ ) with $\mathrm{T}_{1}$ and $\mathrm{T}_{2}$ (unit: $\left.\mathrm{E}_{\mathrm{p}}\right)$ as parameters, as shown in figure 3.

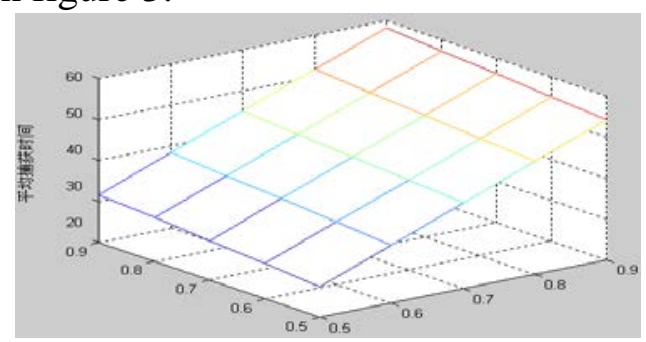

Figure3 average capture time under simulation of Matlab

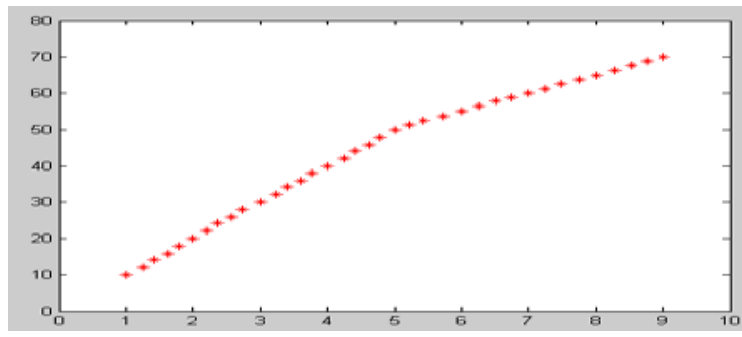

Figure 4 relationship between average capture time and times of repetition

In the fixed condition of $T_{1} 、 T_{2}$ and $G$, the simulation diagram for the relationship between average capture time and times of repetition, as shown in figure 4.

Can be seen from the simulation results, when $G_{0}=1$, the average capture time is minimal. Simulation results confirmed that the high precision TR-UWB synchronization algorithm makes full use of the energy of multiple channel which designed in this paper, realized the synchronization of high precision.Using the signal flow chart and the theory of Markov, analyzed the different threshold value and times of repetition which influence on average capture time,provided the basis to improve the anti-interference ability of communication system in the set pulse bandwidth. 


\section{References}

[1]N.He and C.Tepedelenlioglu,“Adaptive synchronization for noncoherent UWB receivers,” in Proc. IEEE International Conference on Acoustics, Speech, and Signal Processing 2004, vol. 4, pp. 517-520.

[2] R. Hoctor and H. Tomlinson, “Delay-hopped transmitted-reference RF communications,” in Proc. IEEE Conference on Ultra Wideband Systems and Technologies 2002, pp. 265-269.

[3] M. Ho, V. Somayazulu, J. Foerster, and S. Roy, “A differential detector for an ultra-wideband communications system,” in Proc. IEEE 55 ${ }^{\text {th }}$ Vehicular Technology Conference 2002, vol. 4, pp. 1896-1900.

[4] N. He and C. Tepedelenlioglu, "Performance analysis of non-coherent UWB receivers at different synchronization levels,” in Proc. IEEE Global Telecommunications Conference 2004, vol. 6, pp. 3517-3521.

[5] Y. Ma, F. Chin, B. Kannan, and S. Pasupathy, “Acquisition performance of an ultra wide-band communications system over a multiple-access fading channel,” in Proc. IEEE Conference on Ultra Wideband Systems and Technologies 2002, pp. 99-103.

[6] Ning he and Cihan Tepedelenlioglu Joint pulse and symbol level acqusition of uwb receivers IEEE transaction on wireless communication vol 7,pp,6-14NO.1,JANURARY 2008

[7] Rongrongzheng and xiaodaidong Synchronization and integration region optimization for UWB signals with Non-coherent detection and auto-correlation detection. IEEE transaction on communications,VOL.56 pp790-798,MAY 2008 